\title{
SHOCK WAVE VIBRATION FROM A RIVETING HAMMER CAUSES ALTERED SENSORY PERCEPTION AND CUTANEOUS NERVE DAMAGE IN THE RAT-TAIL \\ Sandya Govindaraju ${ }^{1}$, Olaf Rogness ${ }^{2}$, Magnus Persson ${ }^{3}$, James Bain ${ }^{1}$, Danny Riley*1 \\ ${ }^{1}$ Medical College of Wisconsin, Milwaukee, WI-53226 \\ ${ }^{2}$ Milwaukee School of Engineering, Milwaukee, WI 53202 \\ ${ }^{3}$ Atlas Copco Tools, Stockholm, Sweden
}

\section{Introduction}

Peripheral neuropathy is a major component of Hand-arm vibration syndrome (HAVS), an occupational disease affecting workers exposed to vibration from hand held poweredtools. Patients with HAVS complain of persistent tingling, numbness, and sensory dysfunction. Several neurophysiological studies of vibration-exposed patients have reported reduced sensory nerve conduction velocity and amplitude in the median and radial nerves. Altered vibrotactile and temperature thresholds also suggest that vibration may injure peripheral nerve endings and mechanoreceptors ${ }^{1}$. Patients with HAVS show a significant reduction in the number of nerve fibers in skin biopsies ${ }^{2}$. Using the rat-tail vibration model, we investigated the effects on tail skin innervation of shock wave vibration from a riveting hammer activated for $12 \mathrm{~min}$.

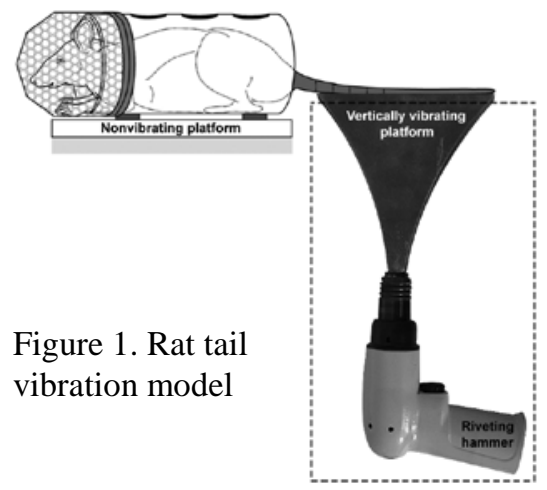

\section{Methods}

Vibration protocol: Sprague-Dawley male rats weighing 275-300 gm were randomly assigned to one of four groups: shock wave day 0 , shock wave 4-day survival, sham day 0 and sham 4-day survival, with an $n$ of 7 or 8 rats/group. The rat was restrained in a tubular cage mounted to a non-vibrating platform. The Atlas Copco riveting gun (RRH04P) was stabilized in a custom-built steel rig. A fan-shaped steel impactor was fabricated as the tool piece to deliver the impulse vibration from the gun and serve as the test platform for the tail (figure 1). The test rat-tails were taped to the platform and vibrated 12 min. The sham animals were restrained with their tails taped to non-vibrating metal platforms within two feet of the riveting hammer.

Tail-flick response: The proximal, middle and distal tail segments were stimulated by a noxious heat test apparatus, and the elapsed time at which the rat flicked the tail from the heat was recorded before and immediately after vibration or sham-vibration exposure. The tail-flick test was repeated on day 2 and day 4 for the rats in the survival groups.

Immunohistochemistry: Cryostat cross sections $(30 \mu \mathrm{m})$ of tail skin were immunostained with pan-neuronal primary rabbit polyclonal antibodies PGP 9.5 (1:4000 in 0.5\% Triton X100, phosphate buffered, Axell, Westbury, NY), goat anti-rabbit biotinylated secondary (1:1000 in 0.5\% Triton phosphate buffer, Invitrogen) and avidin linked Alexa-fluor 488 tertiary (1:1000 in 0.5\% Triton phosphate buffer, Invitrogen). Immunofluorescence photomicrographs were taken using a fixed time exposure for all sections. The intensity of the immunohistochemical signal pixel brightness was measured using MetaMorph 5.2 imaging software (West Chester, PA). 


\section{Results}

Figure 2: The tail flick response times were unchanged through the 4 days in the sham vibration group. The shock vibration group demonstrated a significant 34\% decrease in mean response time immediately following vibration. Two days post-vibration, the response time was not different from the preexposure value. By day 4, the response time was significantly prolonged to $141 \%$ of the

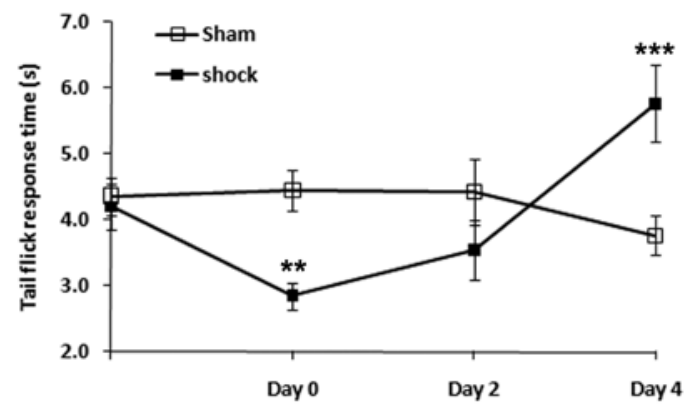
pre-exposure value.
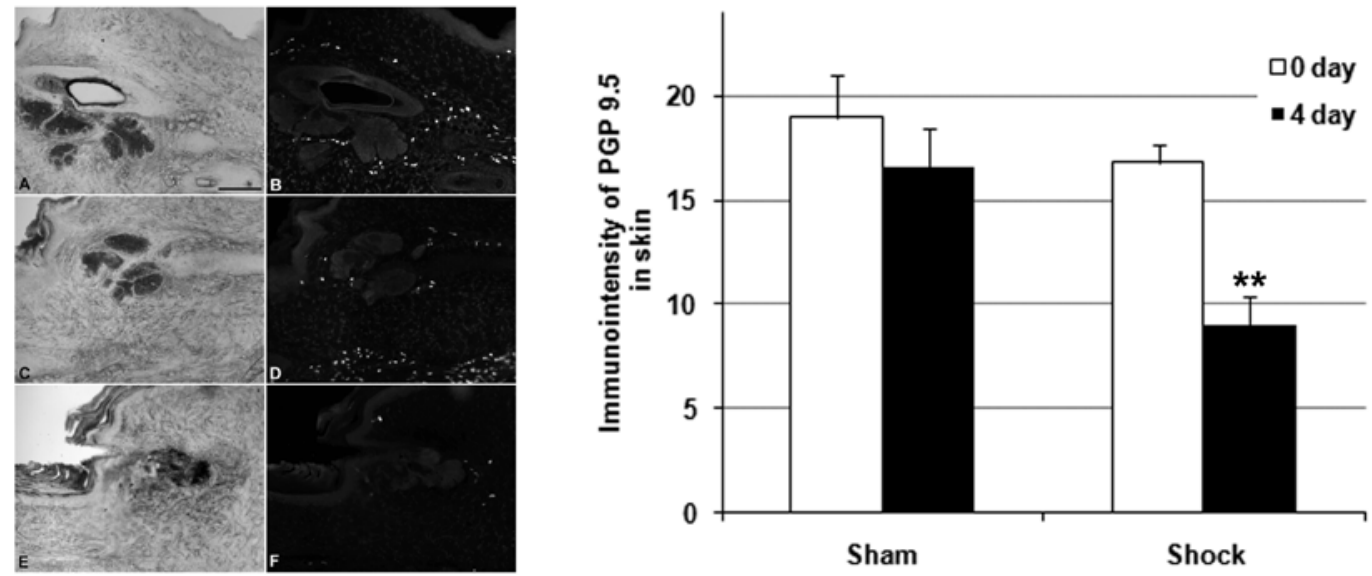

Figures 3 and 4: Tail skin sections from sham day 0 (A brightfield, $\mathrm{B}$ immunofluorescence), shock day 0 (C, D) demonstrated normal immunofluorescence staining of nerve fibers with PGP 9.5 antibody. The shock day 4 group (E,F) exhibited decreased immunoreactivity. ${ }^{* *}$ significantly different from all other groups, $\mathrm{p}<0.01$.

\section{Summary and Conclusions}

1. Shock wave vibration causes a hypersensitization to thermal stimuli on day 0 and hyposensitivity by day 4 .

2. The hyposensitivity is correlated with loss of nerve fibers innervating the tail skin.

3. The striking functional and structural deficits induced by a single, 12-min exposure to shock wave vibration reveal that impulse vibration is highly neuropathological.

\section{References}

1. Stromberg, T., L.B. Dahlin, and G. Lundborg, Vibrotactile sense in the hand-arm vibration syndrome. Scand J Work Environ Health, 1998. 24(6): p. 495-502.

2. Goldsmith, P.C., et al., Cutaneous nerve fibre depletion in vibration white finger. J R Soc Med, 1994. 87(7): p. 377-81. 